\title{
USING ARCHITECTURAL MODELS TO IDENTIFY OPPORTUNITIES FOR IMPROVEMENT OF ACQUISITION MANAGEMENT
}

\author{
Aleksandar DIMOV, Gueorgui STANKOV, and Todor TAGAREV
}

\begin{abstract}
The article presents a model of the processes of one of the Bulgarian force-management subsystems - the acquisition system. The modeling approach adopted is architecture-oriented, which means that the processes are represented according to their sub-processes or phases and activities and the connections in terms of information exchanged between them. The modeling framework selected for this purpose is the widely accepted in NATO and partner countries DODAF (Department of Defense Modeling Framework). The authors provide a brief discussion of the model and some recommendations for improvement of the process of acquisition management.
\end{abstract}

Keywords: Force management, acquisition management, architectural models, DODAF.

\section{Introduction}

Architecture within the terms of the Information Technologies (IT) domain refers to global view organization of a system and its lifecycle, together with its decomposition into subsystems and their interrelations. Generally, architecture could mean either an implementation or a description of the system, i.e. a model.

Although often associated strictly with construction, civil engineering or information technology, this term may also relate more broadly to the practice and optimization of business organizations' internal processes. In this case it is also referred as Enterprise Architecture and addresses organizational business structure, performance management and process architecture. According to Wikipedia, Enterprise Architecture is the practice of applying a comprehensive and rigorous method for describing a current and/or future structure and behavior for an organization's processes, information systems, personnel and organizational sub-units, so that they align with the organization's core goals and strategic direction. ${ }^{1}$ 
From the viewpoint of force management, it is of crucial importance to focus the efforts on research of system architecture as a whole and also on the subsystems. This could improve management of subsystems' processes, because architectural description of processes and systems within an organization enables possibilities for different important analyses and planning of the whole system lifecycle. For example, cost and effort effectiveness assessment, improvement or optimization are activities that should bring to speeding up of a particular process. Another important benefit is the ability to compare and share descriptions about similar architectures across other organizations.

The Bulgarian Force Management System (BFMS) is divided into three main subsystems: Integrated Defense Resources Management System (IDRMS), Required Operational Capabilities (ROC) system and Acquisition system. Currently, BFMS lacks complete and comprehensive description of its architecture. There exists some effort in description of the architecture of some particular warfare subsystems, created within the terms of the Department of Defense Architecture Framework (DODAF). ${ }^{2}$ However, such descriptions do not focus on the highest organizational level of the force management system, but go into specific details and challenges of the described systems (i.e. C4ISR system). In order to be able to analyze and thus, to find gaps and bottlenecks into the structure and the organization of the processes of the Bulgarian Army, a more concise modeling of the force management system is needed.

In this respect, the purpose of the research described in this article is to model within DODAF the process of acquisition management of the Bulgarian military forces. For this purpose we distinguish the relevant acquisition processes and involved organizations and create the so-called operational views of the DODAF framework.

\section{Acquisition Management Processes and Systems}

In general, acquisition refers to analyzing the requirements, development, supplying and disposal of armaments, equipment, services (non-physical products like consultancy, logistics support, training, etc.) and/or infrastructure, in order to satisfy force management needs intended for use in or in support of military missions. ${ }^{3}$ In some cases, a number of acquisition assets is possible to be developed by external, even civil companies or organizations. Although, in most situations, these external organizations are established defense industry suppliers, a clear and competitive contracting procedure is needed in order to ensure best results for the force management system. In general, acquisition considers the whole lifecycle of a force capability support products - from the inception of its requirements to the disposal.

It is widely recognized that contemporary warfare systems should remain in service for as long as possible. Moreover, such systems are often large-scale, which results in 
their high cost and complexity and usually decisions that have been made in early project phases are of crucial importance. These are major reasons for the need of detailed planning and good management of acquisition projects. Such decisions for instance, could be those defining what actually will be acquired, which if not properly made may result in significant shortfall. Acquisition attempts to address these issues by adopting an approach which views the project as a single undertaking across its entire lifecycle from identification of need through to disposal. This way, acquisition includes activities, structured according to the following basic acquisition project stages:

- Identification of the requirements for equipment and/or services to meet army needs;

- Development and/or procurement of equipment and services based on identified requirements;

- Support throughout the equipment/service lifecycle;

- Disposal of unnecessary equipment and/or termination of excess services.

The BFMS Acquisition subsystem does not make an exclusion of this general description. Moreover, it tries to support the capability even after disposal of the product by incorporating rules for initiation of another acquisition project. Further in this article under the term Acquisition or Acquisition System we will mean the BFMS Acquisition subsystem. As a part of BFMS it includes activities for planning, development, testing, logistics, deployment, disposal, etc. of technical systems, equipment, infrastructure, materiel and services all supporting military missions. ${ }^{4}$

The BFMS acquisition system operates in tight connection with the other two subsystems of BFMS: the IDRMS and ROC systems. Its objective is to affordably develop, procure and manage materiel, services and infrastructure to meet validated Mission Needs Statements.

As mentioned earlier in this paper, possible way to improve acquisition system planning is to focus on modeling of its architecture. In the next section, we introduce the notion of architectural model and the methodologies and frameworks for its creation, commonly used in military organizations.

\section{Architectural Models, Modeling Frameworks, and Tools}

As already mentioned, a model of the architecture is needed in order to be able to reason about it in a scientific way. Usually architectural models are created within some predefined framework. It should outline how to organize and present different views that introduce architectural structure and flow of processes within the system. Architectural frameworks are necessary in order to pose the rules for creation of de- 
scriptions in uniform way. This way, a framework facilitates usage of common principles, assumptions and terminology when modeling enterprise architecture. There exists a number of architectural description frameworks such as the Zachman Framework ${ }^{5}$ and the TOGAF framework. ${ }^{6}$ These two are intended for use in general commercial organizations and there also exist similar such frameworks for the defense industry. Most popular of them is the U.S. Department of Defense Architecture Framework (DODAF). ${ }^{7}$ Other frameworks (that are actually based on DODAF) are the UK Ministry of Defence Architecture Framework (MODAF) ${ }^{8}$ and the one developed in France under the name AGATE. ${ }^{9}$

Currently, DODAF is available in version 1.5, developed in April 2007 and defines a common approach for architecture description, development, presentation, and integration for military operations and business processes within the Department of Defense.

Architectures are depicted with different graphic, tabular or purely text products that are logically decomposed into three views: Operational, Systems and Technical and each of them aims to show particular architecture attributes. Final goal is to provide a description framework that enables analysis of the effectiveness and simulation of the processes. As per definition, ${ }^{10}$ Operational View (OV) focuses on behaviors and functions describing the enterprise mission aspects; System View (SV) describes the system and applications supporting mission functions; Technical View (TV) describes the policies, standards and constraints. Additionally, the framework defines the so-called All Views (AV) that contains the overarching information describing the architecture plans, scope, and definitions. Further, each view is presented by several products, which are graphical, tabular and/or textual entities, describing different characteristics and viewpoints of architecture.

A major driving force for development and research on modeling descriptions of Systems Architecture is suitability of models to be manipulated by appropriate software tools. Moreover, an adequate tool support should also help in creation of the models. One of the popular tools that support modeling of many architectural aspects is Telelogic's System Architect. ${ }^{11}$ It offers wide variety of means to model architecture in DODAF. The following section of the paper presents the DODAF model of the BFMS acquisition system built using System Architect.

\section{Architectural Model of the Bulgarian Acquisition Management System}

As mentioned in the introduction, major purpose of this article is to model within DODAF the process of acquisition management of the Bulgarian military forces. More specifically we will show the operational views (OVs) of acquisition products lifecycle management process architecture. The reason for focusing on OVs is that 
when analyzing the model they may show eventual bottlenecks and possibilities for improvement (e.g. optimization by cost, time, effort, etc.) of the process. The OVs presented here are as follows:

- OV-1, which usually contains the high-level operational concept graphical description of the architecture. In some cases, it may also present some textual description.

- OV-2 is the Operational Node Connectivity Description. This architecture view presents operational nodes within the architecture of the acquisition system together with connectivity and information exchange between them.

- OV-5: Operational Activity Model; This view presents capabilities, operational activities, relationships among activities, inputs, and outputs.

- OV-6: describes operational activity and is divided into three sub-views as follows:

- OV-6a is called operational rules model and it identifies business rules that constrain operation;

- OV-6b is called operational state transition description and it identifies business process responses to events;

- OV-6c is called operational event-trace description and it traces actions in a scenario or sequence of events.

According to a definition in normative documents, the BFMS acquisition management process comprises of six phases in the management of products life cycle. They are:

- Concept Exploration and Technology Development

- System Development and Demonstration

- Operational Demonstration, Evaluation, and Selection

- Production or Procurement, Development, and Sustainment

- Product Improvement or Service Life Extension

- Disposal.

\section{Operational View OV-1 Model}

As seen in Figure 1 the high-level structure of BFMS places the acquisition system in between ROC and IDRMS. It neither takes a direct input, nor produces a direct output to the customer. Instead it takes requirements documents (called Mission Needs Statement) from ROC and after coordination with available resources (most often financial) selects the best out of several procurement offers and provides for successful exploitation of the procured resources. 


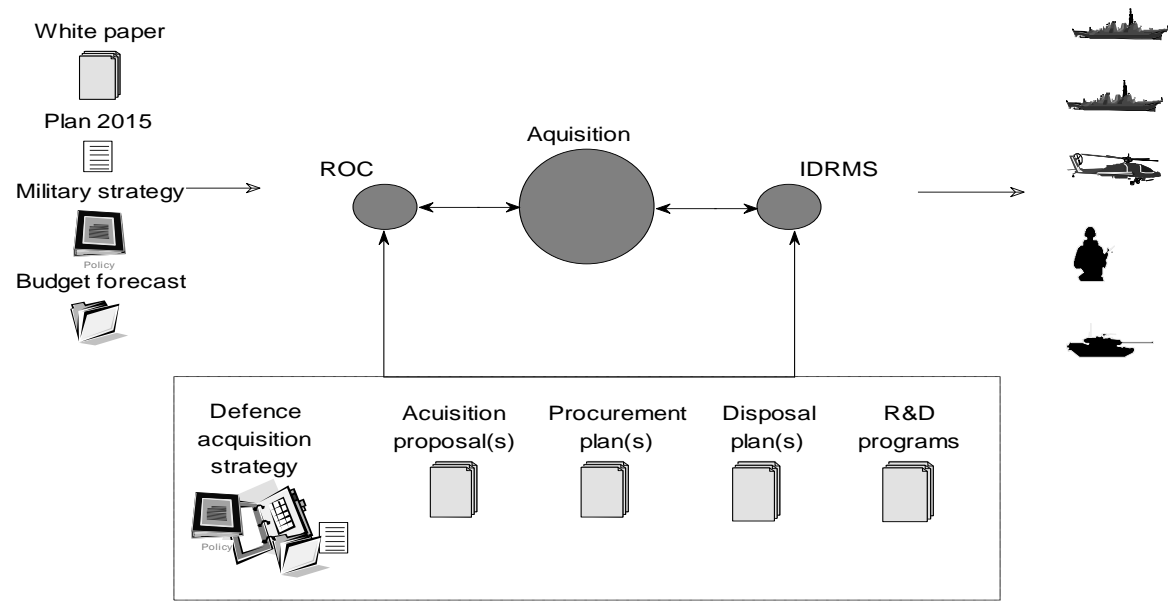

Figure 1: High Level Acquisition Management View Diagram.

\section{Operational Node Connectivity Models}

This view shows the operational nodes in the system and the information exchanges between them. In the context of acquisition projects management, it may be constructed following two different approaches.

Operational nodes are seen as abstract entities, each of which presents one of the phases in the acquisition management process. This approach supposes that operational nodes are groupings of like activities that are performed together to carry out the operational concept. ${ }^{12}$

Operational nodes are purely physical items and each of them represents a single organization involved in the acquisition management process.

The OV-2 created using the first approach is shown in Figure 2 and OV-2 created using the second approach could be seen in Figure 3.

In order to increase the expressiveness of the model, the IDRMS and ROC subsystems are also included in the first case as well as one additional node, named Organizational memory. It should act as a repository of plans developed during the acquisition project that should be used in other projects. For example, a significant plan is the Personnel Management Plan. It may be used to assist force developers in determining the expected number of personnel and in identifying skills and training required during the lifecycle of the acquisition product. 


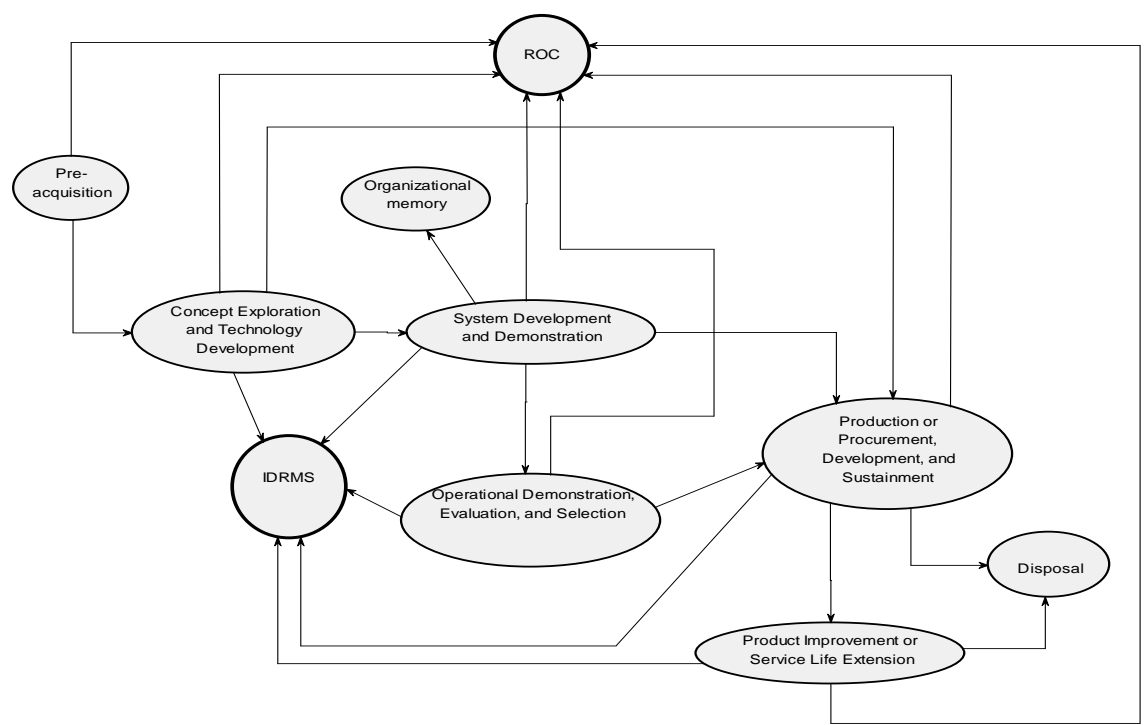

Figure 2: Abstract Operational Node Connectivity Diagram.

\section{Operational View OV-5 Model}

The operational view OV-5 is presented by two diagrams:

- Activity Model Diagram, presented in Figure 4

- Node Tree Diagram that could be seen in Figure 5.

Activity model diagram shows the sequence of workflow actions, together with their required (inputs) and produced (outputs) resources. As seen in the figure, each phase needs specific documents (either completed or working versions) from previous phases and then produces the required documents for the next phases of the acquisition process. Note that phases can also modify working versions of the documents in order to finish them or provide them for further modification until completion. Figure 4 shows that at the start of the procedure of an acquisition project two major documents are needed - Acquisition Decision Memorandum (ADM) and Mission Needs Statement (MNS). MNS comes from the ROC system and defines the basic requirement for the products needed. ADM is issued as permission for every acquisition phase to begin. It is coordinated with both other subsystems - IDRMS and ROC, according to the available resources (IDRMS) and eventual changes in the requirements (ROC). 


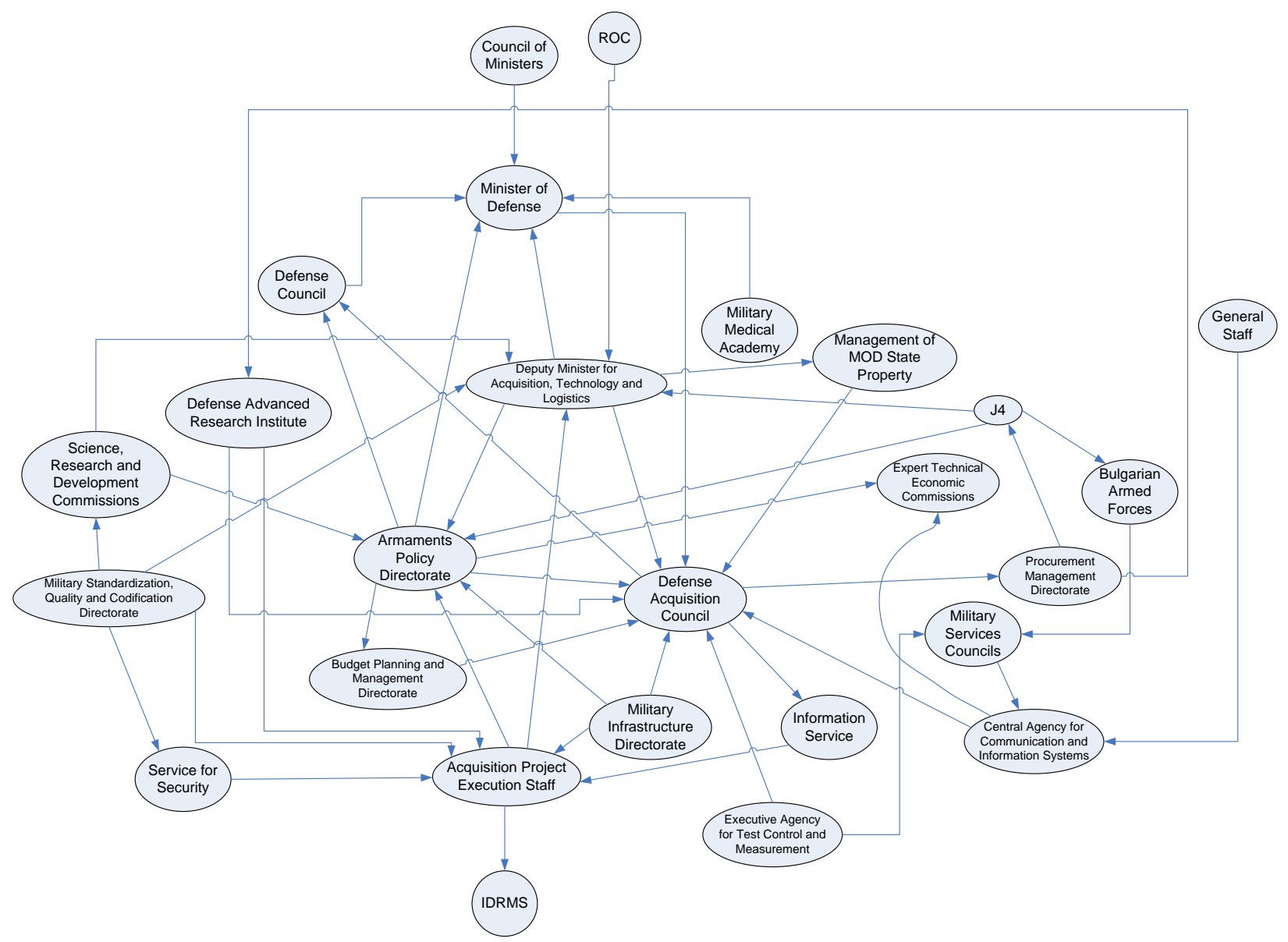

Figure 3: OV-2 Physical Operational Nodes Diagram. 


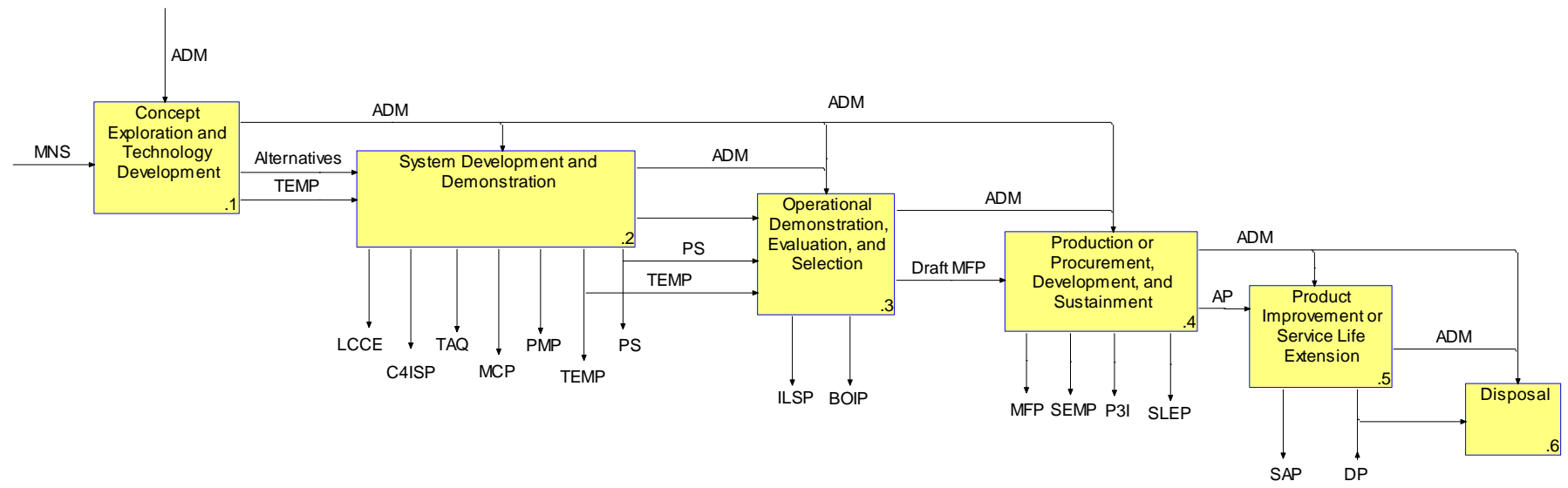

Figure 4: Acquisition Management Activity Model Diagram.

MNS - Mission Needs Statement ADM - Acquisition Decision Memorandum

LCCE - Life-Cycle Cost Estimate

C4ISP - Command, Control, Computers, Communications, and Information System Plan

TAQ - Total Acquisition Quantity

MCP Maintenance Concept Plan

PMP Personnel Management Plan

TEMP Test and Evaluation Management Plan

PS - Procurement Strategy

ILSP - Integrated Logistic Support Plan

BOIP - Basis of Issue Plan

MFP - Materiel Fielding Plan

SEMP - System Engineering Management Plan

P3I - Preplanned Product Improvement Plan

SLEP - Service Life Extension Plan

DP - Disposal Plan 


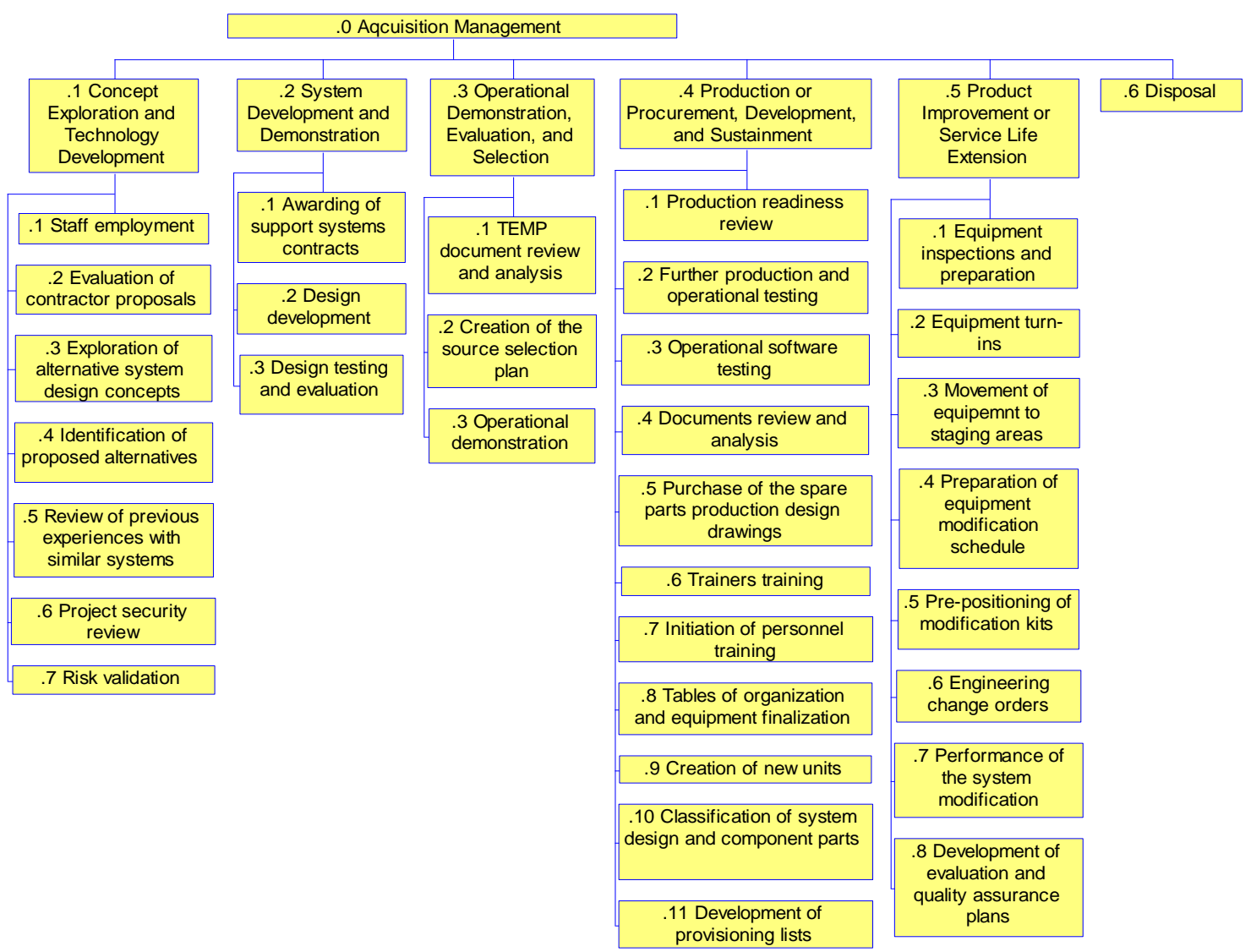

5: Acquisition Management Node Tree Diagram. 


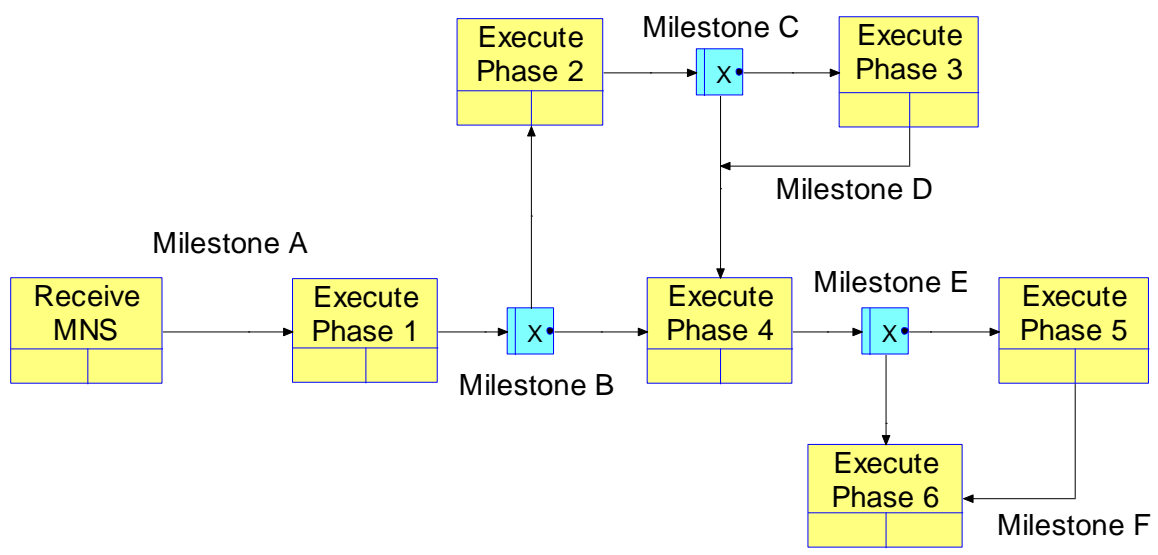

Figure 6: Operational Rules Model.

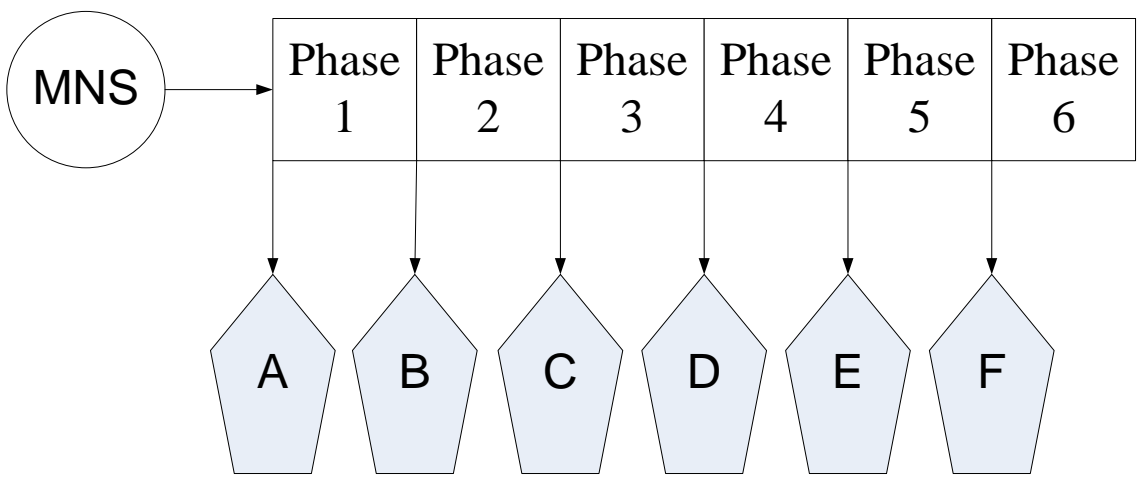

\section{Milestones}

Figure 7: Acquisition Milestones.

The node tree diagram presents the six main acquisition phases and the activities, which they include. The activities of the phases are not included in other diagrams in the article in order to prevent models from overloading and to preserve their understandability. 


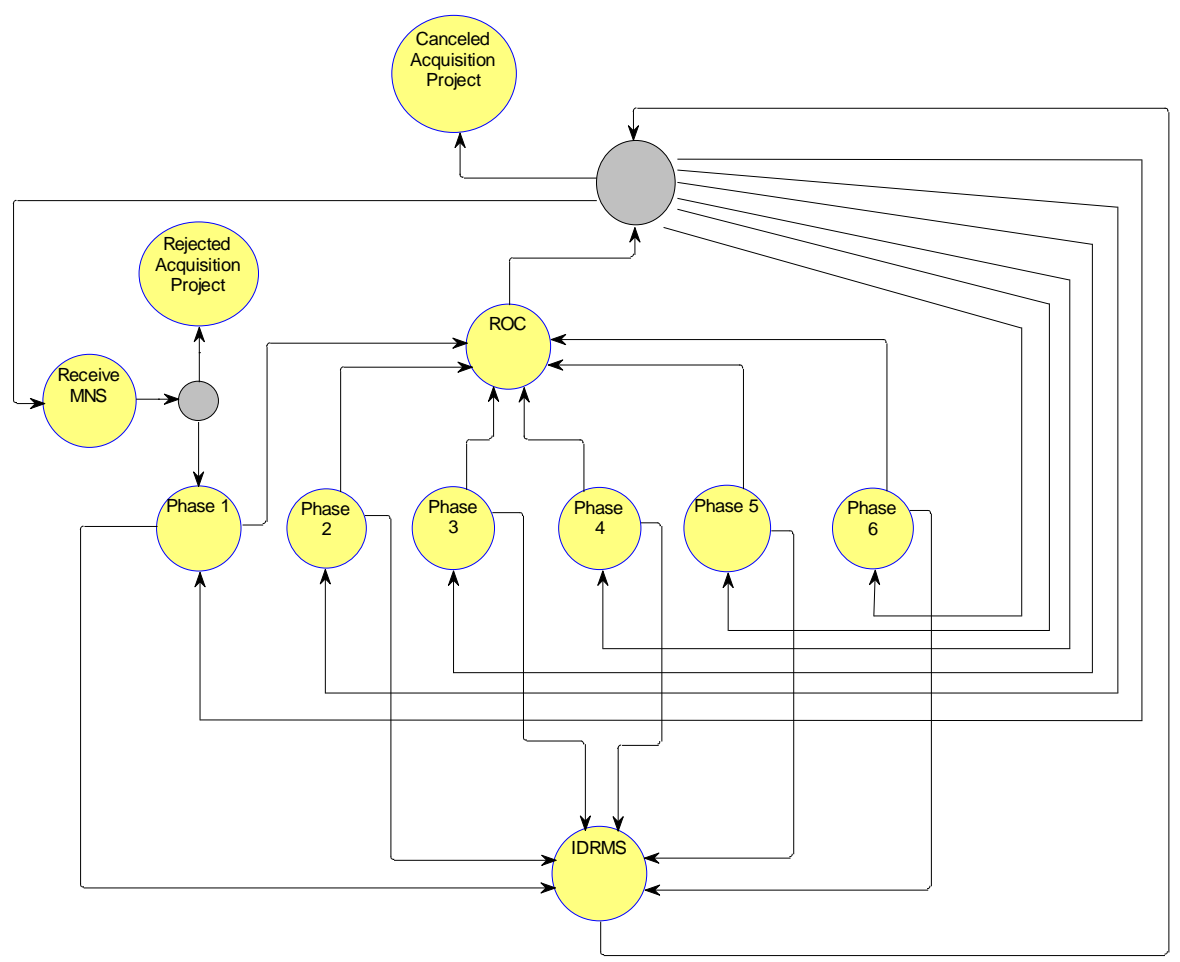

Figure 8: Acquisition Management Operational State Transition Diagram.

The three views of the acquisition management process shown so far present only its structure, i.e. they are static models. The following subsections will present the dynamic aspects of the acquisition management process.

\section{Operational View OV-6a Model}

OV-6a (Figure 6) is the first of a set of three views used to express dynamic behavior (sequence, timing of events and rules for constraining operation) of the architecture. This view sets the constraints of operation of the process. In the particular case with the model of BFMS it shows possible traces for execution of the phases for acquisition of a product. As seen from the figure, at particular stages (the milestones) a decision permits certain phase to be omitted or repeated. The milestones (Figure 7) are the moments when the ADM (see Figure 4) should be released. This view shows the guidelines that should be used further in the improvement of the model of the acquisition architecture. 


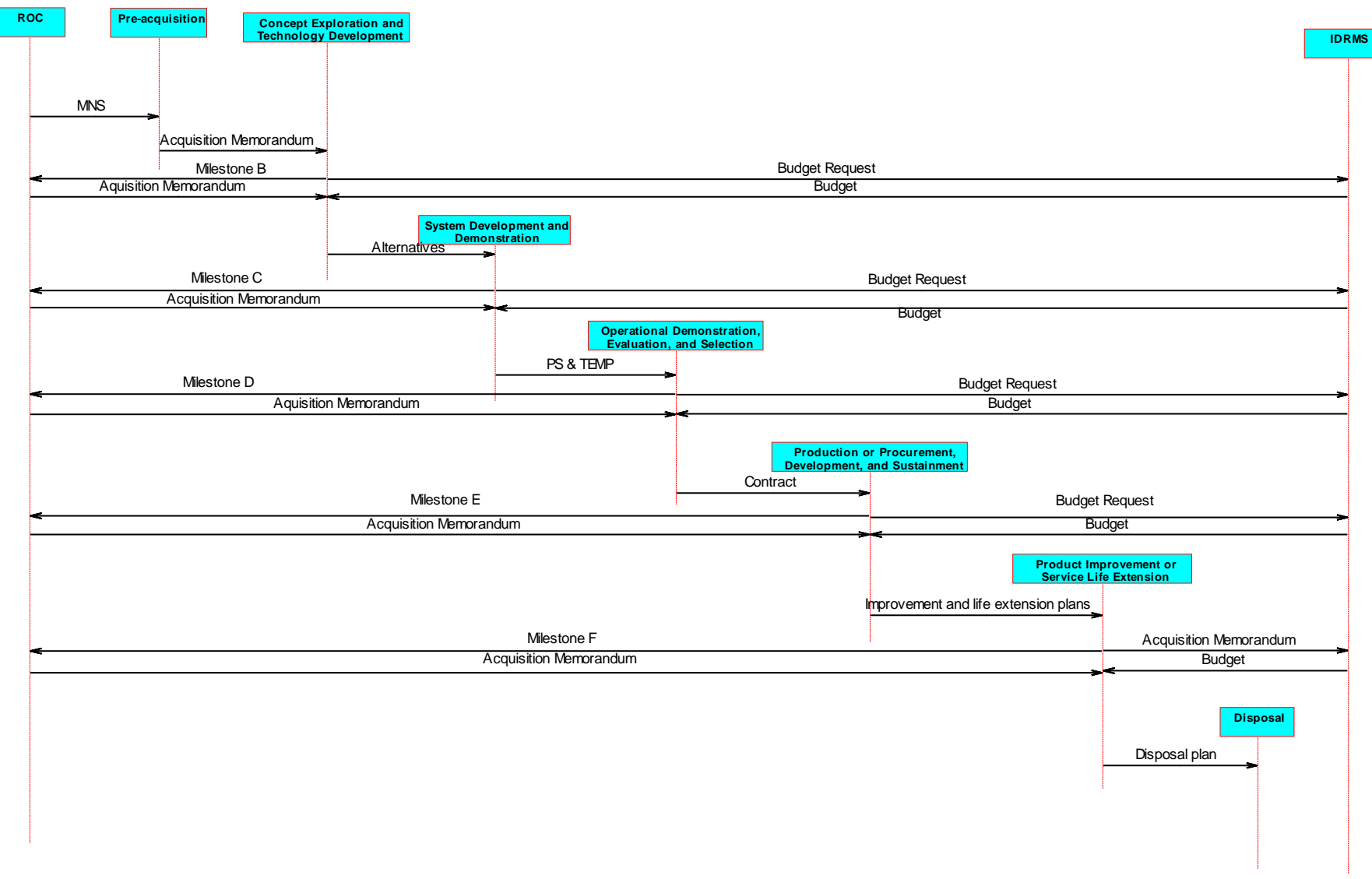

Figure 9: Acquisition Management Operation Event/Trace Diagram. 


\section{Operational View OV-6b Model}

The OV-6b model shown in Figure 8 presents how the whole process changes its states with respect to reaching a particular milestone. The main issue visible from this diagram is that documents and plans created by the acquisition team at each milestone should be used as a basis for decision on whether to continue with the same project, initiate a new one, or cancel it.

\section{Operational View OV-6c Model}

The OV-6c (Figure 9) is actually a time-diagram that shows the possible sequence of events and actions that are taken in response within the process architecture. With respect to OV-6a and OV-6b, the events shown here are most precisely defined. They represent the issuing of actual plans and documents produced at each acquisition phase. In Figure 9 it is possible to trace how completion of particular document influences the next phases in the acquisition process.

\section{Conclusions}

The aim of the article is to build a model of the acquisition process and its activities in the Bulgarian Army Force Management (BFMS). The model shows different aspects of the related activities, together with their relations and the organizations responsible for them. Tools used to build and study the models are Telelogic $\AA^{\prime}$ 's System Architect ${ }^{\circledR}$ and DOORS ${ }$. They allow modeling of multiple abstraction layers of the architecture - business processes, its constituent objects and components and the corresponding data and their structural relationships. As a consequence of this work some statements are inferred aiming to improve the efficiency of acquisition management.

The presented in this article views on the architecture model of the architecture of the BFMS acquisition management system shows some important issues that may be used for optimization of the process. As seen in Figure 3, there are three operational organizations (i.e. nodes) which are significantly overloaded with information exchanges with other participants in the acquisition project. These are the Deputy Minister for Acquisition, Technology and Logistics; the Armaments Policy Directorate and the Defense Acquisition council. Their operational nodes are shown with thick lines in Figure 3. Moreover, the structure of information exchange of the nodes is not strictly hierarchal. Only the Council of Ministers, the Minister of Defense and the Defense Council are hierarchically organized. Other management responsible organizations may receive documents and plans from many levels of BFMS, as seen in Figure 3. In this respect, a possible improvement of the architecture is to reorganize the 
structure of information exchange to be more hierarchical, which will remove some of the overload from the bold-lined organizations in Figure 3.

Another question that arises from the presented models and might require discussion is the presence of phase \#5 (Product Improvement or Service Life Extension) in the process. A closer look at Figure 5 shows that the activities that take place in this phase are to some extent similar to what happens in other phases of acquisition management. This may lead to the conclusion that the fifth phase may be removed from the process, because it actually represents sub-acquisition process. Note that it actually allows a Separate Acquisition Project (SAP) to take place, as shown in Figure 4.

Our directions for further work are two-fold: (1) continuation of the construction of the model and building of the systems and technical DODAF views of acquisition architecture and (2) creation and analysis of improved models of acquisition management.

\section{Notes:}

${ }^{1}$ Enterprise architecture, from Wikipedia <en.wikipedia.org/wiki/Enterprise_architecture> (26 February 2008).

2 Todor Tagarev and Petya Ivanova, "Developing an Architecture for Naval Sovereignty Operations Center," Information \& Security: An International Journal 16 (2005): 29-38.

3 Glossary Defense Acquisition Acronyms and Terms (Fort Belvoir, Virginia: Defense Acquisition University Press, September 2003). 
4 Rules for Management of MoD and Bulgarian Army Defense Products Life Cycle, September 2004 (in Bulgarian), <www.mod.bg/bg/docs/pravilnik_2.html> (26 February 2008).

5 Zachman Framework <apps.adcom.uci.edu/EnterpriseArch/Zachman/> (26 February 2008).

6 TOGAF - The Open Group Architecture Framework, <www.opengroup.org/architecture/ togaf8-doc/arch/> (26 February 2008).

7 DoD Architecture Framework, Definitions and Guidelines, v1.5 <www.defenselink.mil/cionii/docs/DoDAF_Volume_I.pdf> (26 February 2008); DoD Architecture Framework, Product Descriptions, v1.5 <www.defenselink.mil/cio-nii/docs/DoDAF_Volume_II.pdf> (26 February 2008).

8 The official MODAF site, <www.modaf.org.uk/> (26 Feb. 2008).

9 AGATE (architecture framework), from Wikipedia <en.wikipedia.org/wiki/AGATE _(architecture_framework)> (26 February 2008).

${ }^{10}$ DoD Architecture Framework, Definitions and Guidelines; DoD Architecture Framework, Product Descriptions.

${ }^{11}$ Telelogic System Architect, <www.telelogic.com/products/systemarchitect/index.cfm> (26 February 2008).

${ }^{12}$ DoD Architecture Framework, Product Descriptions.

TODOR TAGAREV - Information about the author is available on page 114 of this volume.

ALEKSANDAR DIMOV, $\mathrm{PhD}$, is a researcher at the University of Sofia "St. Cl. Ohridski". $\mathrm{He}$ has received his master's degree in automation engineering from the Technical University of Sofia (2001). He has defended a PhD thesis in the area of description of software architectures in December 2006. His main research interests are in the areas of Software Engineering and Architecture and other related disciplines; Business processes modeling and Project Management.

LTC GUEORGUI STANKOV - Information about the author is available on page 140 of this volume. 